\title{
Existence and Uniqueness of Solutions for a Fractional Order Antiperiodic Boundary Value Problem with a $p$-Laplacian Operator
}

\author{
Ruihui Huang \\ Department of Mathematics and Statistics, Wuhan University, Wuhan, Hubei 430072, China
}

Correspondence should be addressed to Ruihui Huang; huang_ruihui@163.com

Received 20 June 2013; Accepted 19 November 2013

Academic Editor: Yong Hong Wu

Copyright (C) 2013 Ruihui Huang. This is an open access article distributed under the Creative Commons Attribution License, which permits unrestricted use, distribution, and reproduction in any medium, provided the original work is properly cited.

We study the existence and uniqueness of solutions for a class of antiperiodic boundary value problems of the fractional differential equation with a $p$-Laplacian operator. Based on the Leray-Schauder nonlinear alternative, several sufficient conditions of the existence and uniqueness of solution of the above problem are established. Our results improve and complement the recent work of Chen and Liu, 2012.

\section{Introduction}

Economics is a rich source for mathematical ideas. Particularly, mathematical model is an important tool designed to describe the operation of the economy of a country or a region. Such model is usually created to examine the dynamics of quantities as, for instance, level of prices, unemployment, total income, total amount of goods and services produced, investment demand, and so on. Recently, many mathematical models of differential equations are used for fitting the available economic data. Of course, many of the economic models are static, but there are also some dynamic models, describing the economy over the time periods such as GDP or employment, while the differential equations relating these variables are intended to describe economic decisions. Because fractional operators are nonlocal, they are more suitable for constructing models possessing memory effect with the long time periods, and then fractional differential equations possess large advantage in describing economic phenomena over the time periods. So it is important to investigate the underlying economic models of fractional differential equations theoretically in order to get an in-depth understanding of the systems and then make scientific economic decisions [1-5].

Several theoretical interesting results are available in the literature [6-14] for the existence and uniqueness of solution of fractional order differential equations. In [6], by using the iterative technique, Zhang and Han investigated the existence and uniqueness of positive solutions for the fractional differential model with integral boundary conditions, and some growth conditions were adopted to guarantee the existence of unique positive solution for fractional differential model. Again the iterative technique is applied by Zhang et al. [12] to obtain the iterative solutions of a nonlinear fractional differential equations. Besides the iterative technique, the Banach contraction mapping principle is also a main tool to get the existence and uniqueness of solution. Recently, Leibenson [15] introduced the $p$-Laplacian equation to describe a turbulent flow in a porous medium in mechanics problem:

$$
\left(\varphi_{p}\left(x^{\prime}(t)\right)\right)^{\prime}=f\left(t, x(t), x^{\prime}(t)\right)
$$

where $\varphi_{p}(s)=|s|^{p-2} s, p>1$. Motivated by Leibenson's work, Chen and Liu [13] investigated the existence of solutions for the antiperiodic boundary value problem of a fractional $p$ Laplacian equation with the following form (for short ABVP):

$$
\begin{aligned}
& \mathscr{D}_{0+}^{\beta} \varphi_{p}\left(\mathscr{D}_{0+}^{\alpha} x(t)\right)=f(t, x(t)), \quad t \in[0,1], \\
& x(0)=-x(1), \quad \mathscr{D}_{0+}^{\alpha} x(0)=-\mathscr{D}_{0+}^{\alpha} x(1),
\end{aligned}
$$


where $0<\alpha, \beta \leq 1,1<\alpha+\beta \leq 2, \mathscr{D}_{0+}^{\alpha}$ is a Caputo fractional derivative, and $f:[0,1] \times \mathbb{R}^{2} \rightarrow \mathbb{R}$ is continuous. By using Schaefer's fixed point theorem, the authors established the following result.

Theorem 1. Let $f:[0,1] \times \mathbb{R} \rightarrow \mathbb{R}$ be continuous. Assume that

$(H)$ there exist nonnegative functions $a, b \in C[0,1]$ such that

$$
|f(t, u)| \leq a(t)+b(t)|u|^{p-1}, \quad \forall t \in[0,1], u \in \mathbb{R} .
$$

Then ABVP (2) has at least one solution, provided that

$$
\frac{3^{q}\|b\|_{\infty}^{q-1}}{2^{q} \Gamma(\alpha+1)(\Gamma(\beta+1))^{q-1}}<1,
$$

where $q$ satisfies $1 / p+1 / q=1$.

The aim of this paper is to improve and complement the work of Chen and Liu. Firstly, we introduce the following definitions which are used in our main results.

Definition 2. A function $\psi:(-\infty,+\infty) \rightarrow[0,+\infty)$ is called a $C-N$ function if it is a continuous nondecreasing function. Again if $\psi$ satisfies $\psi(r)<r, r>0$, then $\psi$ is called a nonlinear $\mathscr{D}$-contraction mapping.

Definition 3. A function $f$ is said to be a Crathéodory's function if the following conditions hold:

(i) for each $x \in \mathbb{R}$, the mapping $t \mapsto f(t, x)$ is Lebesgue measurable.

(ii) for a.e. $t \in[0,1]$, the mapping $x \mapsto f(t, x)$ is continuous on $\mathbb{R}$.

Let us finish our presentation to announce our main results that can be stated as follows:

Theorem 4. Let $f$ be a Crathéodory's function. Assume that

$(S)$ there exist some constant $\epsilon \in(0, \beta)$ such that $a(t)$, $b(t) \in L^{1 / \epsilon}([0,1],[0,+\infty))$ and $a C-N$ function $\psi$ with

$$
|f(t, x)| \leq a(t)+b(t) \psi(|x|), \quad \text { a.e. }(t, x) \in[0,1] \times \mathbb{R} .
$$

Then the ABVP (2) has at least one solution, provided that there exists a real number $r>0$ such that

$\frac{3}{2 \Gamma(\alpha+1)}\left[\frac{3}{2 \Gamma(\beta)}\left(\frac{1-\epsilon}{\beta-\epsilon}\right)^{1-\epsilon}\left(\|a\|_{L^{1 / \epsilon}}+\|b\|_{\left.L^{1 / \epsilon} \psi(r)\right)}\right]^{q-1} \leq r\right.$.

The following some cases are straightforward consequences of Theorem 4; the proof can follow as Theorem 4 and we will omit the corresponding proof.
Corollary 5. Let $f$ be a Crathéodory's function. Assume that

$\left(S^{*}\right)$ there exists some constant $\epsilon \in(0, \beta)$ such that $a(t) \in L^{1 / \epsilon}([0,1],[0,+\infty))$ and

$$
|f(t, x)| \leq a(t), \quad \text { a.e. }(t, x) \in[0,1] \times \mathbb{R} .
$$

Then the ABVP (2) has at least one solution, provided that there exists a real number $r>0$ such that

$$
\frac{3}{2 \Gamma(\alpha+1)}\left[\frac{3}{2 \Gamma(\beta)}\left(\frac{1-\epsilon}{\beta-\epsilon}\right)^{1-\epsilon}\|a\|_{L^{1 / \epsilon}}\right]^{q-1} \leq r .
$$

Corollary 6. Let $f$ be a Crathéodory's function. Assume that

$\left(S^{* *}\right)$ there exists some constant $\epsilon \in(0, \beta)$ such that $b(t)$ $\in L^{1 / \epsilon}([0,1],[0,+\infty))$ and $a C-N$ function $\psi$ with

$$
|f(t, x)| \leq b(t) \psi(|x|), \quad \text { a.e. }(t, x) \in[0,1] \times \mathbb{R} \text {. }
$$

Then the ABVP (2) has at least one solution, provided that there exists a real number $r>0$ such that

$$
\frac{3}{2 \Gamma(\alpha+1)}\left[\frac{3}{2 \Gamma(\beta)}\left(\frac{1-\epsilon}{\beta-\epsilon}\right)^{1-\epsilon}\|b\|_{L^{1 / \epsilon} \psi(r)}\right]^{q-1} \leq r .
$$

Remark 7. The main results of this paper improve the work of Chen and Liu from the following three aspects.

(1) In [13], a stronger condition that $f:[0,1] \times \mathbb{R} \rightarrow \mathbb{R}$ is continuous is required, but in this paper we only require that $f$ satisfies Crathéodory's condition, which is a weaker condition than those of paper [13].

(2) In Theorem $4, a(t), b(t) \in L^{1 / \epsilon}([0,1],[0,+\infty))$ for some $\epsilon \in(0, \beta)$, and $a, b$ can be singular at some zero measure set of $[0,1]$. However, $a(t), b(t)$ are continuous in paper [13], which are not allowed to have singularity in $[0,1]$.

(3) In the main results of this paper, $\psi$ is only required to be a $C-N$ function. Clearly, a $C-N$ function includes $\psi(|u|)=|u|^{p-1}, p>1$ as special case.

In what follows, we also complement a uniqueness result on the ABVP (2), which is based on the Banach contraction mapping principle and a basic property of the $p$-Laplacian operator: if $q>2,|x|,|y| \leq M$, then

$$
\left|\varphi_{q}(x)-\varphi_{q}(y)\right| \leq(q-1) M^{q-2}|x-y| .
$$

In the case $1<p<2$, due to $1 / p+1 / q=1$, we can get $q>2$.

Theorem 8. Let $f$ be a Crathéodory's function. Assume that $1<p<2$ and

$(\widehat{S})$ there exist some constant $\epsilon \in(0, \beta)$ such that $a(t)$, $b(t) \in L^{1 / \epsilon}([0,1],[0,+\infty))$ and $a$ D-contraction mapping $\psi$ with

$$
\begin{array}{r}
|f(t, x)| \leq a(t), \quad|f(t, x)-f(t, y)| \leq b(t) \psi(|x-y|), \\
\text { a.e. }(t, x) \in[0,1] \times \mathbb{R} .
\end{array}
$$


Then the ABVP (2) has a unique solution, provided that

$$
\frac{3(q-1)}{2 \Gamma(\alpha+1)}\left[\frac{3}{2 \Gamma(\beta)}\left(\frac{1-\epsilon}{\beta-\epsilon}\right)^{1-\epsilon}\right]^{q-1}\|a\|_{L^{1 / \epsilon}}^{q-2}\|b\|_{L^{1 / \epsilon}}=: \kappa<1 .
$$

\section{Preliminaries and Lemmas}

For the convenience of the reader, we present here some necessary basic knowledge and definitions about fractional calculus theory which are to be used in the later sections.

Definition 9 (see [16]). The Riemann-Liouville fractional integral operator of order $\alpha>0$ of a function $x:(a,+\infty) \rightarrow$ $R$ is given by

$$
I_{0+}^{\alpha} x(t)=\frac{1}{\Gamma(\alpha)} \int_{a}^{t}(t-s)^{\alpha-1} x(s) d s
$$

provided that the right side integral is pointwise defined on $(a,+\infty)$.

Definition 10 (see [16]). The Caputo fractional derivative of order $\alpha>0$ of a continuous function $x:(a,+\infty) \rightarrow R$ is given by

$$
\mathscr{D}_{0+}^{\alpha} x(t)=\frac{1}{\Gamma(n-\alpha)} \int_{a}^{t}(t-s)^{n-\alpha-1} x^{(n)}(s) d s,
$$

where $n$ is the smallest integer greater than or equal to $\alpha$, provided that the right side integral is pointwise defined on $(a,+\infty)$.

Proposition 11 (see [17]). Let $\alpha>0$. Assume that $x, \mathscr{D}_{0+}^{\alpha} x \in$ $L^{1}(0,1)$. Then the following equality holds:

$$
I_{0+}^{\alpha} \mathscr{D}_{0+}^{\alpha} x(t)=x(t)+c_{0}+c_{1} t+c_{2} t^{2}+\cdots+c_{n} t^{n-1},
$$

where $c_{i} \in \mathbb{R}(i=0,1,2, \ldots, n-1), n$ is the smallest integer greater than or equal to $\alpha$.

As a consequence of Proposition 11, Chen and Liu obtained the following lemma.

Lemma 12 (see [13]). Given $h \in C[0,1]$, the unique solution of

$$
\begin{gathered}
\mathscr{D}_{0+}^{\beta} \varphi_{p}\left(\mathscr{D}_{0+}^{\alpha} x(t)\right)=h(t), \quad t \in[0,1], \\
x(0)=-x(1), \quad \mathscr{D}_{0+}^{\alpha} x(0)=-\mathscr{D}_{0+}^{\alpha} x(1)
\end{gathered}
$$

is

$$
\begin{aligned}
x(t)=\frac{1}{\Gamma(\alpha)} \int_{0}^{t}(t-s)^{\alpha-1} \varphi_{q}( & \frac{1}{\Gamma(\beta)} \int_{0}^{s}(s-\tau)^{\beta-1} h(\tau) d \tau \\
& +A h(s)) d s+B h(t),
\end{aligned}
$$

where

$$
A h(t)=-\left.\frac{1}{2} I_{0+}^{\beta} h(t)\right|_{t=1}=-\frac{1}{2 \Gamma(\beta)} \int_{0}^{1}(1-s)^{\beta-1} h(s) d s,
$$

$\forall t \in[0,1]$,

$B h(t)$

$$
\begin{aligned}
= & -\left.\frac{1}{2} I_{0+}^{\alpha} \varphi_{q}\left(\frac{1}{2} I_{0+}^{\beta} h(t)+A h(t)\right)\right|_{t=1} \\
= & -\frac{1}{2 \Gamma(\alpha)} \int_{0}^{1}(1-s)^{\alpha-1} \varphi_{q} \\
& \times\left(\frac{1}{\Gamma(\beta)} \int_{0}^{s}(s-\tau)^{\beta-1} h(\tau) d \tau+A h(s)\right) d s,
\end{aligned}
$$

$\forall t \in[0,1]$.

As [13], define the Nemytskii operator $N: C[0,1] \rightarrow$ $C[0,1]$ as follows:

$$
N x(t)=f(t, x(t)), \quad t \in[0,1],
$$

and denote a nonlinear operator $F: C[0,1] \rightarrow C[0,1]$ by

$$
\begin{aligned}
& F x(t)=I_{0+}^{\alpha} \varphi_{q}\left(I_{0+}^{\beta} N x(t)+A N x(t)\right)+B N x(t) \\
& =\frac{1}{\Gamma(\alpha)} \int_{0}^{t}(t-s)^{\alpha-1} \varphi_{q} \\
& \times\left(\frac{1}{\Gamma(\beta)} \int_{0}^{s}(s-\tau)^{\beta-1} f(\tau, x(\tau)) d \tau\right. \\
& -\frac{1}{2 \Gamma(\beta)} \int_{0}^{1}(1-\tau)^{\beta-1} \\
& \times f(\tau, x(\tau)) d \tau) d s \\
& -\frac{1}{2 \Gamma(\alpha)} \int_{0}^{1}(1-s)^{\alpha-1} \varphi_{q} \\
& \times\left(\frac{1}{\Gamma(\beta)} \int_{0}^{s}(s-\tau)^{\beta-1} f(\tau, x(\tau)) d \tau\right. \\
& -\frac{1}{2 \Gamma(\beta)} \int_{0}^{1}(1-\tau)^{\beta-1} \\
& \times f(\tau, x(\tau)) d \tau) d s
\end{aligned}
$$

then the fixed points of the operator $F$ are solutions of the ABVP (2).

Our main tool is based on the following known LeraySchauder nonlinear alternative theorem.

Lemma 13. Let $X$ be a real Banach space and let $\Omega$ be a bounded open subset of $X$, where $\theta \in \Omega, T: \bar{\Omega} \rightarrow X$ 
is a completely continuous operator. Then, either there exists $x \in \partial \Omega, \lambda>1$ such that $T x=\lambda x$, or there exists a fixed point $x^{*} \in \bar{\Omega}$.

\section{The Proof of the Main Results}

Proof of Theorem 4. Consider the operator $F: C[0,1] \rightarrow$ $C[0,1]$ defined by $(21)$. For the sake of convenience, we subdivide the proof into two steps.

Step 1. $F: C[0,1] \rightarrow C[0,1]$ is completely continuous.

Let $\Omega \subset C[0,1]$ be any bounded set. We will prove that $F(\Omega)$ is also bounded. In fact, for any $x \in \Omega$, there exists a $l>0$ such that $\|x\| \leq l$, and then by the Hölder inequality

$$
\begin{aligned}
& \left|I_{0+}^{\beta} N x(t)+A N x(t)\right| \\
& =\mid \frac{1}{\Gamma(\beta)} \int_{0}^{s}(s-\tau)^{\beta-1} f(\tau, x(\tau)) d \tau \\
& -\frac{1}{2 \Gamma(\beta)} \int_{0}^{1}(1-\tau)^{\beta-1} f(\tau, x(\tau)) d \tau \mid \\
& \leq \frac{1}{\Gamma(\beta)} \int_{0}^{s}(s-\tau)^{\beta-1}|f(\tau, x(\tau))| d \tau \\
& +\frac{1}{2 \Gamma(\beta)} \int_{0}^{1}(1-\tau)^{\beta-1}|f(\tau, x(\tau))| d \tau \\
& \leq \frac{1}{\Gamma(\beta)} \int_{0}^{s}(s-\tau)^{\beta-1} \\
& \times(a(\tau)+b(\tau) \psi(|x(\tau)|)) d \tau \\
& +\frac{1}{2 \Gamma(\beta)} \\
& \times \int_{0}^{1}(1-\tau)^{\beta-1}(a(\tau)+b(\tau) \psi(|x(\tau)|)) d \tau \\
& \leq \frac{1}{\Gamma(\beta)}\left(\frac{1-\epsilon}{\beta-\epsilon}\right)^{1-\epsilon}\left(\|a\|_{L^{1 / \epsilon}}+\|b\|_{L^{1 / \epsilon}} \psi(l)\right) \\
& +\frac{1}{2 \Gamma(\beta)}\left(\frac{1-\epsilon}{\beta-\epsilon}\right)^{1-\epsilon}\left(\|a\|_{L^{1 / \epsilon}}+\|b\|_{L^{1 / \epsilon}} \psi(l)\right) \\
& =\frac{3}{2 \Gamma(\beta)}\left(\frac{1-\epsilon}{\beta-\epsilon}\right)^{1-\epsilon} \\
& \times\left(\|a\|_{L^{1 / \epsilon}}+\|b\|_{L^{1 / \epsilon}} \psi(l)\right)=: L, \\
& =\mid \frac{1}{2 \Gamma(\alpha)} \int_{0}^{1}(1-s)^{\alpha-1} \varphi_{q}
\end{aligned}
$$

$$
\begin{gathered}
\times\left(\frac{1}{\Gamma(\beta)} \int_{0}^{s}(s-\tau)^{\beta-1} f(\tau, x(\tau)) d \tau\right. \\
-\frac{1}{2 \Gamma(\beta)} \int_{0}^{1}(1-\tau)^{\beta-1} \\
\times f(\tau, x(\tau)) d \tau) d s \mid \\
\leq \frac{1}{2 \Gamma(\alpha)} \int_{0}^{1}(1-s)^{\alpha-1} \varphi_{q}(L) d s=\frac{\varphi_{q}(L)}{2 \alpha \Gamma(\alpha)} \\
=\frac{\varphi_{q}(L)}{2 \Gamma(\alpha+1)}
\end{gathered}
$$

It follows from (22) and (23) that for any $t \in[0,1]$

$$
\begin{aligned}
|F x(t)| & =\left|I_{0+}^{\alpha} \varphi_{q}\left(I_{0+}^{\beta} N x(t)+A N x(t)\right)+B N x(t)\right| \\
& \leq \frac{3 \varphi_{q}(L)}{2 \Gamma(\alpha+1)},
\end{aligned}
$$

which implies that $F(\Omega)$ is bounded.

On the other hand, by the continuity of $f$ on $x$ and the Lebesgue dominated convergence theorem, we can get that $F$ is continuous. Moreover, according to the strategy in [13], we know that $F(\Omega) \subset C[0,1]$ is equicontinuous. Thus AscoliArzela theorem assures that $F$ is completely continuous.

Step 2. $F$ has at least a fixed point.

Now consider $B_{r}=\{x \in C[0,1]:\|x\| \leq r\}$. An application of Leray-Schauder nonlinear alternative theorem yields either that the operator $F x=x$ has a fixed point or there exists $x \in \partial B_{r}$ such that $F x=\lambda x$ for some $\lambda>1$. We show that the latter assertion does not hold. Assume the contrary, then there exist a $x \in \partial B_{r}$ and some $\lambda>1$ such that $F x=\lambda x$. By (22)-(24) and (6), we have

$$
\begin{aligned}
\lambda r= & \lambda\|x\|=\|F x\| \\
\leq & \frac{3}{2 \Gamma(\alpha+1)} \varphi_{q} \\
& \times\left(\frac{3}{2 \Gamma(\beta)}\left(\frac{1-\epsilon}{\beta-\epsilon}\right)^{1-\epsilon}\left(\|a\|_{L^{1 / \epsilon}}+\|b\|_{L^{1 / \epsilon}} \psi(r)\right)\right) \\
= & \frac{3}{2 \Gamma(\alpha+1)} \\
& \times\left(\frac{3}{2 \Gamma(\beta)}\left(\frac{1-\epsilon}{\beta-\epsilon}\right)^{1-\epsilon}\left(\|a\|_{L^{1 / \epsilon}}+\|b\|_{\left.L^{1 / \epsilon} \psi(r)\right)}\right)^{q-1}\right. \\
\leq & r,
\end{aligned}
$$

which yields $\lambda \leq 1$, and then a contraction to the inequality $\lambda>1$ is obtained. In consequence, the operator $F x=x$ has a fixed point in $C[0,1]$ with $\|x\| \leq r$. This further implies that the ABVP (2) has at least a solution on $[0,1]$. The proof is completed. 
Proof of Theorem 8. According to (12) and a similar strategy as Theorem 4, we know that $F$ is completely continuous operator. Now we will prove that $F$ is a contraction mapping. By (22), we have

$$
\begin{aligned}
& \left|I_{0+}^{\beta} N x(t)+A N x(t)\right| \\
& \leq \frac{1}{\Gamma(\beta)} \int_{0}^{s}(s-\tau)^{\beta-1}|f(\tau, x(\tau))| d \tau \\
& +\frac{1}{2 \Gamma(\beta)} \int_{0}^{1}(1-\tau)^{\beta-1}|f(\tau, x(\tau))| d \tau \\
& \leq \frac{1}{\Gamma(\beta)} \int_{0}^{s}(s-\tau)^{\beta-1} a(\tau) d \tau+\frac{1}{2 \Gamma(\beta)} \\
& \times \int_{0}^{1}(1-\tau)^{\beta-1} a(\tau) d \tau \\
& \leq \frac{1}{\Gamma(\beta)}\left(\frac{1-\epsilon}{\beta-\epsilon}\right)^{1-\epsilon}\|a\|_{L^{1 / \epsilon}} \\
& +\frac{1}{2 \Gamma(\beta)}\left(\frac{1-\epsilon}{\beta-\epsilon}\right)^{1-\epsilon}\|a\|_{L^{1 / \epsilon}} \\
& =\frac{3}{2 \Gamma(\beta)}\left(\frac{1-\epsilon}{\beta-\epsilon}\right)^{1-\epsilon}\|a\|_{L^{1 / \epsilon}}=: M\|a\|_{L^{1 / \epsilon}}, \\
& \left|I_{0+}^{\beta} N x(t)+A N x(t)-I_{0+}^{\beta} N y(t)-A N y(t)\right| \\
& \leq \frac{1}{\Gamma(\beta)} \int_{0}^{s}(s-\tau)^{\beta-1}|f(\tau, x(\tau))-f(\tau, y(\tau))| d \tau \\
& +\frac{1}{2 \Gamma(\beta)} \int_{0}^{1}(1-\tau)^{\beta-1}|f(\tau, x(\tau))-f(\tau, y(\tau))| d \tau \\
& \leq \frac{1}{\Gamma(\beta)} \int_{0}^{s}(s-\tau)^{\beta-1} b(\tau) \psi(|x-y|) d \tau \\
& +\frac{1}{2 \Gamma(\beta)} \int_{0}^{1}(1-\tau)^{\beta-1} b(\tau) \psi(|x-y|) d \tau \\
& \leq \frac{1}{\Gamma(\beta)}\left(\frac{1-\epsilon}{\beta-\epsilon}\right)^{1-\epsilon}\|b\|_{L^{1 / \epsilon} \psi}(\|x-y\|) \\
& +\frac{1}{2 \Gamma(\beta)}\left(\frac{1-\epsilon}{\beta-\epsilon}\right)^{1-\epsilon}\|b\|_{L^{1 / \epsilon} \psi}(\|x-y\|) \\
& =\frac{3}{2 \Gamma(\beta)}\left(\frac{1-\epsilon}{\beta-\epsilon}\right)^{1-\epsilon}\|b\|_{L^{1 / \epsilon}}\|x-y\| \\
& =M\|b\|_{L^{1 / \epsilon}}\|x-y\| \text {. }
\end{aligned}
$$

So it follows from (11)-(12) and (26) that

$$
\begin{aligned}
& |F x(t)-F y(t)| \\
& =\mid I_{0+}^{\alpha} \varphi_{q}\left(I_{0+}^{\beta} N x(t)+A N x(t)\right)+B N x(t) \\
& \quad-I_{0+}^{\alpha} \varphi_{q}\left(I_{0+}^{\beta} N y(t)+A N y(t)\right)-B N y(t) \mid
\end{aligned}
$$

$$
\begin{aligned}
\leq & \mid I_{0+}^{\alpha}\left(\varphi_{q}\left(I_{0+}^{\beta} N x(t)+A N x(t)\right)\right. \\
& \left.\quad-\varphi_{q}\left(I_{0+}^{\beta} N y(t)+A N y(t)\right)\right) \mid \\
\leq & \frac{1}{\Gamma(\alpha)} \int_{0}^{t}(t-s)^{\alpha-1}(q-1) M^{q-1}\|a\|_{L^{1 / \epsilon}}^{q-2}\|b\|_{L^{1 / \epsilon}}\|x-y\| d s \\
& +\frac{1}{2 \Gamma(\alpha)} \int_{0}^{1}(1-s)^{\alpha-1}(q-1) M^{q-1} \\
\leq & \frac{3(q-1)}{2 \Gamma(\alpha+1)}\left[\frac{3}{2 \Gamma(\beta)}\left(\frac{1-\epsilon}{\beta-\epsilon}\right)^{1-\epsilon}\right]^{q-1} \\
& \times\|a\|_{L^{1 / \epsilon}}^{q-2}\|b\|_{L^{1 / \epsilon}}\|x-y\|,
\end{aligned}
$$

which implies that

$$
\|F x-F y\| \leq \kappa\|x-y\|,
$$

and then $F: C[0,1] \rightarrow C[0,1]$ is a contraction mapping since $0<\kappa<1$. By means of the Banach contraction mapping principle, we get that $F$ has a unique fixed point in $C[0,1]$; that is, the $\operatorname{ABVP}(2)$ has a unique solution.

\section{Examples}

In this section we give two examples to illustrate our main results.

Example 1. Consider the following ABVP for the fractional p-Laplacian equation:

$$
\begin{gathered}
\mathscr{D}_{0+}^{1 / 2} \varphi_{3}\left(\mathscr{D}_{0+}^{3 / 4} x(t)\right)=-\frac{1}{10 t^{1 / 8}}+\frac{1}{16 e(1-t)^{1 / 6}} e^{|x(t)|}, \\
t \in[0,1], \\
x(0)=-x(1), \quad \mathscr{D}_{0+}^{3 / 4} x(0)=-\mathscr{D}_{0+}^{3 / 4} x(1) .
\end{gathered}
$$

Corresponding to the ABVP (2), we get that $p=3, q=3 / 2$, $\alpha=3 / 4, \beta=1 / 2$, and

$$
f(t, x)=-\frac{1}{10 t^{1 / 8}}+\frac{1}{8(1-t)^{1 / 6}} \frac{e^{|x|}}{2 e} .
$$

Choose

$$
a(t)=\frac{1}{10 t^{1 / 8}}, \quad b(t)=\frac{1}{5(1-t)^{1 / 6}}, \quad \psi(|x|)=\frac{e^{|x|}}{2 e}
$$

and $\epsilon=1 / 4, r=1$; then

$$
|f(t, x)| \leq a(t)+b(t) \psi(|x|) .
$$


By a simple calculation, we can obtain that

$$
\|a\|_{L^{4}}=\frac{1}{10} \times 2^{1 / 4}, \quad\|b\|_{L^{4}}=\frac{1}{5} \times 3^{1 / 4}, \quad \psi(1)=\frac{1}{2} .
$$

Thus

$$
\begin{aligned}
& \frac{3}{2 \Gamma(\alpha+1)}\left[\frac{3}{2 \Gamma(\beta)}\left(\frac{1-\epsilon}{\beta-\epsilon}\right)^{1-\epsilon}\left(\|a\|_{L^{1 / \epsilon}}+\|b\|_{L^{1 / \epsilon}} \psi(r)\right)\right]^{q-1} \\
& \quad=1.6321 \times\left[0.8463 \times 3^{3 / 4} \times\left(\frac{1}{10} \times 2^{1 / 4}+\frac{1}{5} \times 3^{1 / 4} \times \frac{1}{2}\right)\right]^{3 / 2} \\
& =0.5054<1=r .
\end{aligned}
$$

The above facts imply that the ABVP (1) satisfies all assumptions of Theorem 4. Hence, it has at least a solution.

Example 2. Consider the following ABVP for the fractional p-Laplacian equation:

$$
\begin{aligned}
& \mathscr{D}_{0+}^{1 / 2} \varphi_{3 / 2}\left(\mathscr{D}_{0+}^{3 / 4} x(t)\right)=\frac{\sin x(t)}{10 t^{1 / 8}}, \quad t \in[0,1], \\
& x(0)=-x(1), \quad \mathscr{D}_{0+}^{3 / 4} x(0)=-\mathscr{D}_{0+}^{3 / 4} x(1) .
\end{aligned}
$$

Corresponding to the $\operatorname{ABVP}(2)$, we get that $p=3 / 2, q=3$, $\alpha=3 / 4, \beta=1 / 2$, and

$$
f(t, x)=\frac{\sin x}{10 t^{1 / 8}}
$$

Choose

$$
a(t)=b(t)=\frac{1}{10 t^{1 / 8}}, \quad \psi(|x|)=|x|,
$$

and $\epsilon=1 / 4$; then

$$
\begin{aligned}
|f(t, x)| \leq a(t)= & \frac{1}{10 t^{1 / 8}}, \\
|f(t, x)-f(t, y)| & \leq \frac{1}{10 t^{1 / 8}}|\sin x-\sin y| \\
& \leq b(t) \psi(|x-y|) .
\end{aligned}
$$

By a simple calculation, we can obtain that

$$
\|a\|_{L^{4}}=\|b\|_{L^{4}}=\frac{1}{10} \times 2^{1 / 4}
$$

Thus

$$
\begin{aligned}
& \frac{3(q-1)}{2 \Gamma(\alpha+1)}\left[\frac{3}{2 \Gamma(\beta)}\left(\frac{1-\epsilon}{\beta-\epsilon}\right)^{1-\epsilon}\right]^{q-1}\|a\|_{L^{1 / \epsilon}}^{q-2}\|b\|_{L^{1 / \epsilon}} \\
& =3.2642 \times\left[0.8463 \times 3^{3 / 4}\right]^{2} \times\left(\frac{1}{10} \times 2^{1 / 4}\right)^{2}=0.6171 \\
& =\kappa<1 .
\end{aligned}
$$

The above facts imply that the ABVP (35) satisfies all assumptions of Theorem 8 . Hence, it has a unique solution.
In the end of this work we also remark that Example 1 cannot be solved by using theorem of [13] because $\psi(u)$ is exponential growth and $a(t), b(t)$ are singular at $t=0$ and $t=1$, respectively. We also anticipate that the result of uniqueness here can be extended to the case $p \geq 2$ as an open problem.

\section{References}

[1] T. Škovránek, I. Podlubny, and I. Petráš, "Modeling of the national economies in state-space: a fractional calculus approach," Economic Modelling, vol. 29, no. 4, pp. 1322-1327, 2012.

[2] M. Dalkir, "Spurious correlation under fractional integration in output series," Economics Letters, vol. 107, no. 2, pp. 165-168, 2010.

[3] W. Zhang, We. Xiao, and C. He, "Equity warrants pricing model under fractional brownian motion and an empirical study," Expert Systems with Applications, vol. 36, no. 2, part 2, pp. 3056$3065,2009$.

[4] N. J. Cutland, P. E. Kopp, and W. Willinger, "Stock price returns and the Joseph effect: a fractional version of the Black-Scholes model," in Progress in Probability, vol. 36, pp. 327-351, 1995.

[5] P. Cheridito, "Arbitrage in fractional Brownian motion models," Finance and Stochastics, vol. 7, no. 4, pp. 533-553, 2003.

[6] X. Zhang and Y. Han, "Existence and uniqueness of positive solutions for higher order nonlocal fractional differential equations," Applied Mathematics Letters, vol. 25, no. 3, pp. 555-560, 2012.

[7] C. S. Goodrich, "Existence of a positive solution to a class of fractional differential equations," Applied Mathematics Letters, vol. 23, no. 9, pp. 1050-1055, 2010.

[8] X. Zhang, L. Liu, and Y. Wu, "The uniqueness of positive solution for a singular fractional differential system involving derivatives," Communications in Nonlinear Science and Numerical Simulation, vol. 18, no. 6, pp. 1400-1409, 2013.

[9] C. S. Goodrich, "Existence of a positive solution to systems of differential equations of fractional order," Computers \& Mathematics with Applications, vol. 62, no. 3, pp. 1251-1268, 2011.

[10] X. Zhang, L. Liu, and Y. Wu, "Multiple positive solutions of a singular fractional differential equation with negatively perturbed term," Mathematical and Computer Modelling, vol. 55, no. 3-4, pp. 1263-1274, 2012.

[11] L. S. Leibenson, "General problem of the movement of a compressible fluid in a porous medium," Izvestiya Akademii Nauk Kirgizskoi SSR, vol. 9, pp. 7-10, 1983 (Russian).

[12] X. Zhang, L. Liu, Y. Wu, and Y. Lu, "The iterative solutions of nonlinear fractional differential equations," Applied Mathematics and Computation, vol. 219, no. 9, pp. 4680-4691, 2013.

[13] T. Chen and W. Liu, "An anti-periodic boundary value problem for the fractional differential equation with a $p$-Laplacian operator," Applied Mathematics Letters, vol. 25, no. 11, pp. 1671-1675, 2012.

[14] X. Zhang, L. Liu, B. Wiwatanapataphee, and Y. Wu, "Positive solutions of eigenvalue problems for a class of fractional differential equations with derivatives," Abstract and Applied Analysis, vol. 2012, Article ID 512127, 16 pages, 2012.

[15] X. Zhang, L. Liu, and Y. Wu, "The eigenvalue problem for a singular higher order fractional differential equation involving fractional derivatives," Applied Mathematics and Computation, vol. 218, no. 17, pp. 8526-8536, 2012.

[16] I. Podlubny, Fractional Differential Equations, Academic Press, San Diego, Calif, USA, 1999.

[17] A. A. Kilbas, H. M. Srivastava, and J. J. Trujillo, Theory and Applications of Fractional Differential Equations, vol. 204, Elsevier Science B.V., Amsterdam, The Netherlands, 2006. 


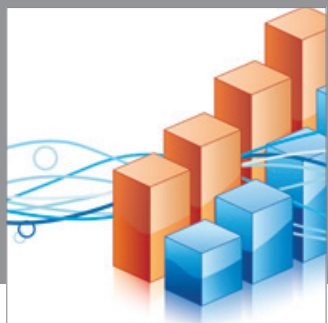

Advances in

Operations Research

mansans

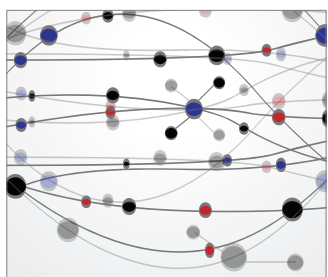

The Scientific World Journal
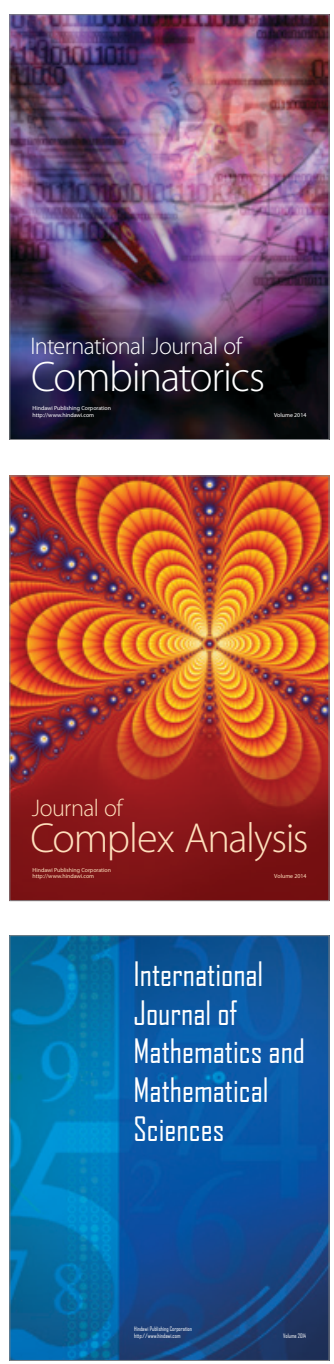
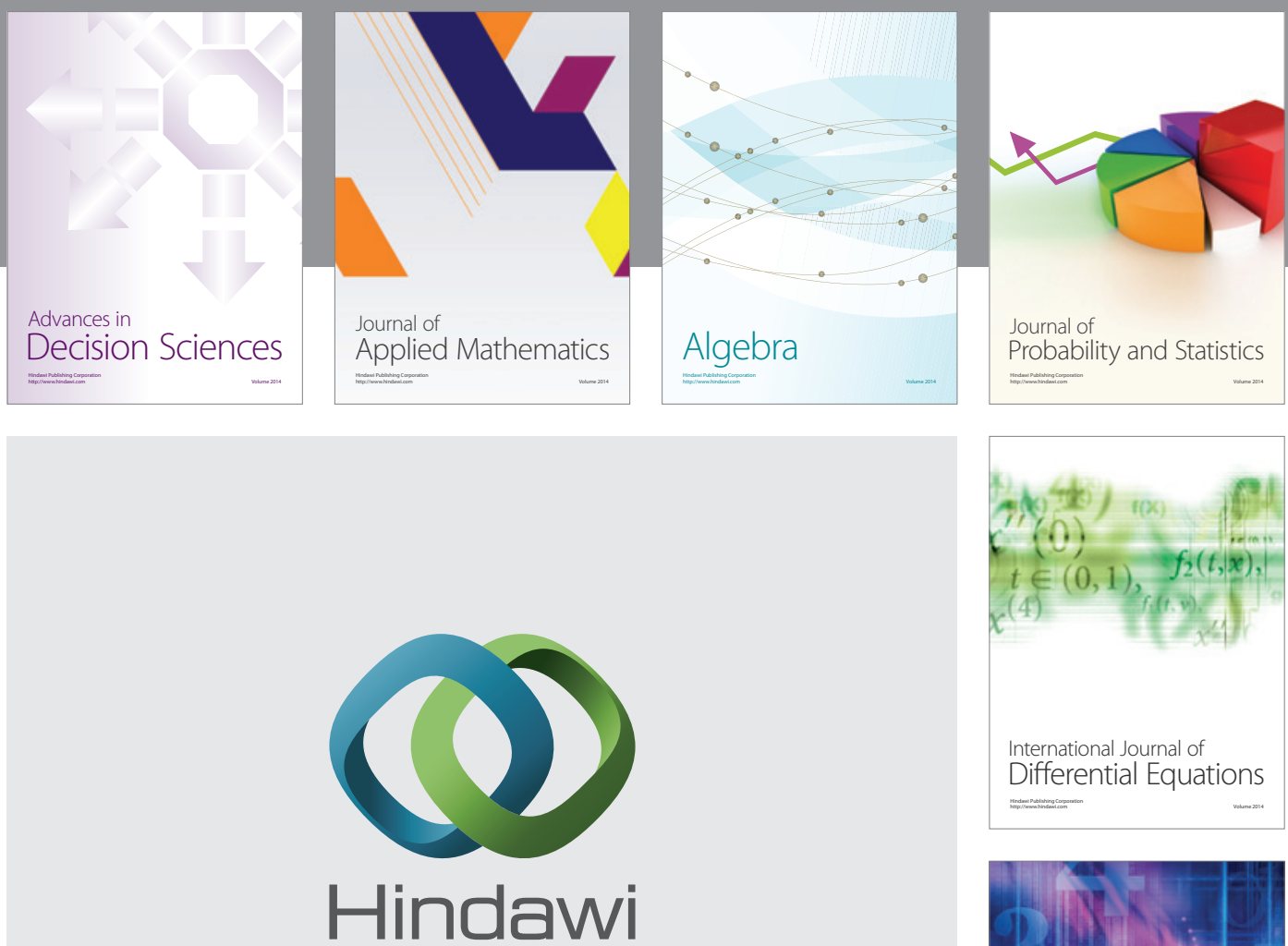

Submit your manuscripts at http://www.hindawi.com
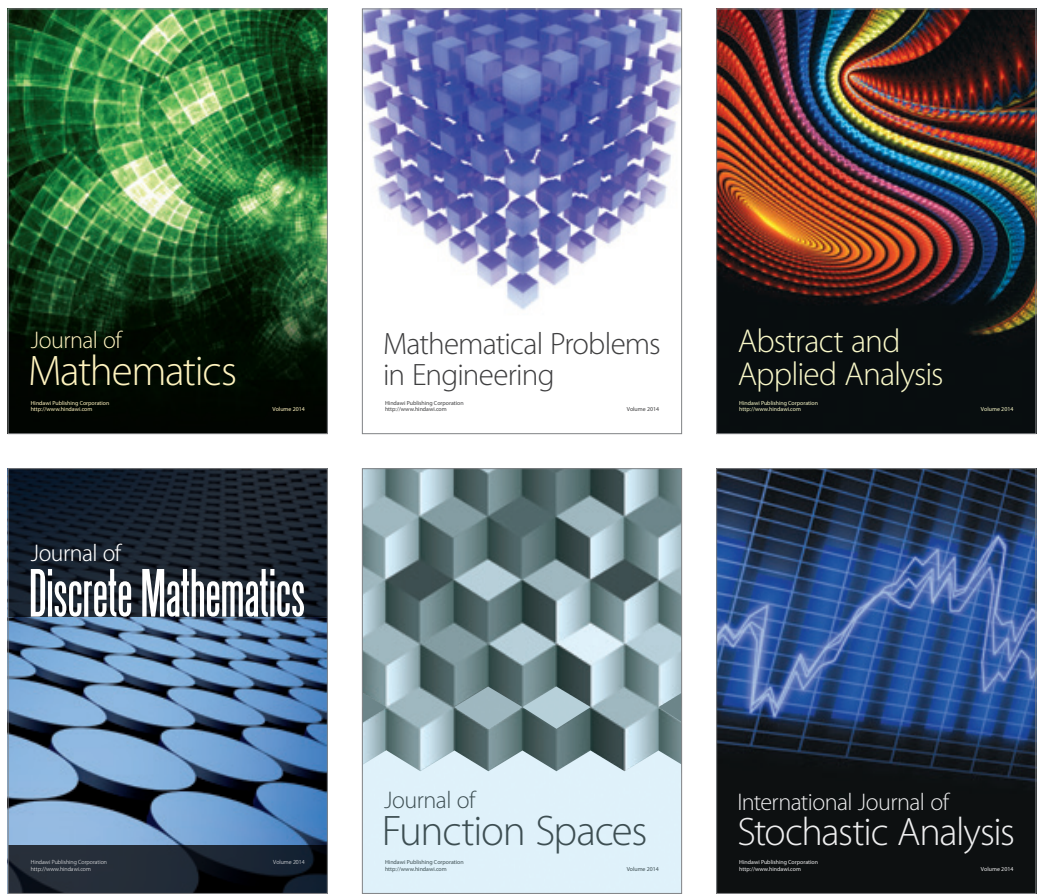

Journal of

Function Spaces

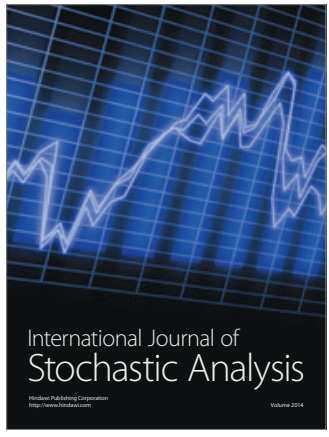

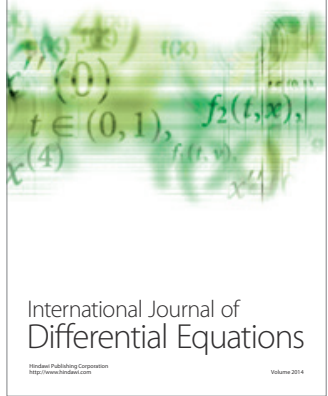
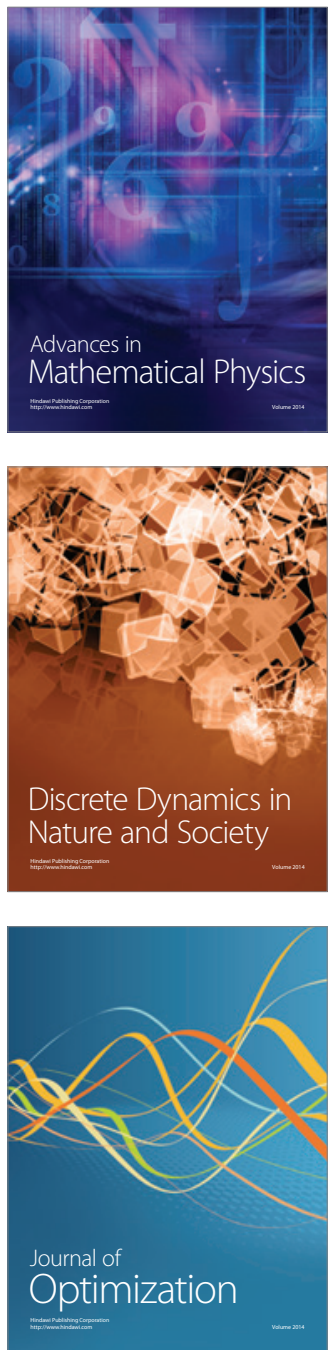